\title{
Gianni Celati e Giorgio Morandi
}

Linea, luce, lontananza

\section{Marco Antonio Bazzocchi}

\section{(2) OpenEdition}

Journals

Edizione digitale

URL: https://journals.openedition.org/cher/677

DOI: $10.4000 /$ cher.677

ISSN: 2803-5992

\section{Editore}

Presses universitaires de Strasbourg

\section{Edizione cartacea}

Data di pubblicazione: 9 juillet 2020

Paginazione: 45-55

ISBN: 979-10-344-0068-3

ISSN: 1968-035X

\section{Notizia bibliografica digitale}

Marco Antonio Bazzocchi, «Gianni Celati e Giorgio Morandi», reCHERches [Online], 24 | 2020, online dal 20 septembre 2021, consultato il 18 novembre 2021. URL: http://journals.openedition.org/cher/677 ; DOI: https://doi.org/10.4000/cher.677 


\title{
Gianni Celati e Giorgio Morandi Linea, luce, lontananza
}

\author{
Marco Antonio BaZZOCCHI
}

$\mathrm{T}$ ra il 1984 e il 1985 possiamo collocare la svolta dell'opera di Gianni Celati nella direzione di una testualità non più legata ai motivi degli anni Settanta ma diretta verso nuove esplorazioni. Narratori delle pianure e Verso la foce sono i due libri che ne derivano. Possiamo ipotizzare che Celati concentri ora la sua attenzione verso elementi del narrare che hanno molta affinità con concetti legati alla percezione visiva: la linea bassa, il limite, la soglia, il bordo, il dentro e il fuori. Sono concetti riconducibili all'incontro con Luigi Ghirri (1981), ai testi brevi che Celati compone evocando la poetica dell'amico fotografo e che arrivano fino a quel bellissimo scritto che è Viaggio in Italia con 20 fotografi, 20 anni dopo. La recensione del 1987 a Palomar di Calvino è il punto di approdo in cui si sentono i risultati di questi anni di meditazione. Lì si parla esplicitamente dell'esterno come oggetto insondabile e dei mezzi elaborati dall'uomo per catturarlo, con immagini, parole, categorie, strumenti. Celati invece propone un rapporto con l'esterno fondato su un esercizio quasi stoico di "calma osservazione» (Celati 1987b: 236). Le parole servono solo come sonde delicate che ci aiutano mentre ci addentriamo nello spazio esterno, "per fissare qualche punto riconoscibile» (Celati 1987b: 236). Dunque le usiamo per descrivere il mondo, consapevoli che non sono rappresentazioni ma solo limiti progressivi dai quali sporgiamo il nostro sguardo sull'esterno. Descriviamo, cioè creiamo linee che corrispondono a bordi e limiti. Oltre i quali sentiamo esserci il vuoto. Ed è al vuoto che tende la nostra attenzione. Per entrare in questo stato particolare del corpo e della mente dobbiamo lasciarci dietro le spalle tutte le categorie con cui abitualmente spieghiamo il mondo.

Ogni limite a cui arriviamo, ogni parola-limite, segna un livello diverso di intensità nel nostro sentire il mondo. Nello scritto del 1984, «Finzioni a cui credere», Celati inizia a parlare di Ghirri mettendolo in rapporto con l'ambiente in cui il fotografo vive, un quartiere della provincia emiliana nel paese di

1 Marco Antonio Bazzocchi, Università di Bologna. 
Formigine. La descrizione di questo quartiere anomalo vuole essere del tutto corrispondente alla definizione del tipo particolare di sguardo che Ghirri ha introdotto nel mondo della finzione. A differenza degli scrittori avidi di catturare le cose, Ghirri è riuscito ad abbassare al massimo la soglia di intensità che gli serve per entrare in rapporto con l'esterno. Le villette geometrili del quartiere in cui abita vengono da lui fotografate mettendo in evidenza regolarità di linee, simmetrie, colori, con vedute frontali che provocano un pensare-immaginare non meno degno di attenzione di quello che richiedono monumenti insigni. Per quanto riguarda la tecnica prospettica e i colori, Celati fa il nome di Piero della Francesca, cioè del pittore che più di tutti ha rappresentato il mondo come insieme di forme astratte, riducendo anche la presenza dell'uomo a un insieme di composizioni geometriche e facendo del colore una componente in perfetta sintonia con la forma. Roberto Longhi (nel famoso saggio del 1927) aveva detto: «sintesi prospettica di forma e colore». Una formula che possiamo spiegare così: Piero delinea figure che sono raccolte dentro la nettezza del contorno, ma il contorno non è altro che il limite a cui arriva un colore per poi lasciar spazio al colore confinante, in modo tale che dal rapporto tra colori emerga anche la distinzione dei piani prospettici. Ogni figura è così definita ma vibra grazie alle risonanze del colore che la mettono in rapporto con tutti i livelli del visibile. (Il colore è vibrazione, la linea stabilità, ma c'è bisogno della linea per creare la vibrazione del rapporto dei colori). Il nome di Piero della Francesca fa pensare a una tradizione pittorica che prende i fatti eccezionali della storia e li porta il più possibile verso il quotidiano, creando spesso rapporti molto intensi tra la presenza dell'uomo, il paesaggio e gli oggetti, secondo una genealogia artistica che risale all'arte del Nord, al mondo fiammingo che Piero aveva conosciuto a Venezia.

Per Celati, abbassare la soglia di intensità significa eliminare il fasto del racconto come ricerca dell'eccezionale o di un meraviglioso artificiale. Celati fa l'esempio di una foto di Ghirri dove si vedono ragazzi nel buio su una spiaggia, illuminati da un'insegna. In questo mondo si creano delle risonanze che ci permettono di capire l'esterno perché «il nostro pensare è già all'esterno». Chi osserva non lo fa seguendo elaborati schemi mentali ma si sente "già da sempre e per sempre» nella rappresentazione. Cosa significa quel "già da sempre»? Dobbiamo partire dall'idea della soglia di intensità per capirlo. Se la soglia è alta, e l'intento è «voler vedere (o descrivere) l'eccezionale», si deve ricorrere «a temi sensazionali», che corrispondono, nelle foto «ad aggressioni visive». Se invece la soglia è bassa (secondo l'operazione condotta da Ghirri) noi siamo in un certo senso «dentro» la rappresentazione, cioè non sentiamo né il bisogno né la difficoltà di varcare un limite (cioè sentiamo appena la presenza di una linea). Dal punto di vista della storia culturale, lo studioso Jonathan Cray ci spiega che questo fenomeno può essere collocato all'altezza della pittura romantica, con Turner per esempio, che rappresenta un mondo in dissoluzione, senza più la necessità dello strumento prospettico per fare in modo che la mente possa concepirlo: «In maniera apparentemente inaspettata, i dipinti eseguiti da Turner 
negli ultimi anni Trenta e negli anni Quaranta dell'Ottocento, sono sintomatici di un'irrevocabile perdita della fonte di luce fissa, della dissoluzione del cono di raggi luminosi e del collasso della distanza che separava l'osservatore dal luogo dell'esperienza ottica» (Cray 2013: 144). Fino al 700 la mente ha bisogno di concepirsi mentre prende le distanze dal mondo, e solo così può rappresentare il mondo. È la stampa di Dürer scelta da Calvino come copertina di Palomar, in cui un diaframma quadrettato si frappone tra l'artista e la modella distesa, come appunto un modello mentale necessario per arrivare alla rappresentazione disegnata. Con alcuni pittori romantici questo sdoppiamento viene superato, la mente è dentro al mondo, lo assorbe e ne viene assorbita. Questo fenomeno avviene perché c'è una nuova consapevolezza della luce e del rapporto tra la luce e le forme, e nello stesso tempo si tiene in considerazione la presenza del vuoto tra gli oggetti. Le forme riempite di luce e di colore vengono chiamate da Celati «apparenze», che significa non semplicemente ciò che appare ma ciò che appare a un occhio che ha rinunciato a conoscere ma vuole solo trovare un posto nell'insieme delle cose del mondo: «a questo punto le apparenze, che sono il supporto della rappresentazione esterna, ci stanno a cuore più d'ogni interpretazione complessiva del mondo: infatti sono tutto ciò che abbiamo per orientarci nello spazio» (Celati 1984: 13). Celati pensa a tre livelli diversi della realtà: da una parte c'è qualcuno che cerca di mettere insieme le proprie esperienze, di fronte a lui ci sono le apparenze del mondo, intorno c'è il vuoto al quale bisogna trovare rimedio quotidiano. Mettere insieme le apparenze, senza perdersi nel vuoto, è quanto secondo Celati ogni essere umano fa, costruendo di volta in volta il proprio «racconto dell'esterno». Ecco che cosa è una «finzione» a cui credere, cioè una finzione che ci si può portar dietro per far fronte al quotidiano e per non cadere nel tranello dell'eccezionale e del fuori luogo.

«Pensare-immaginare» è un doppio uso della mente che Celati ritrova nell'ultimo personaggio di Calvino, il perplesso signor Palomar che viene letto attraverso una idea di Merleau-Ponty, la prosa del mondo. Dove ritorna fuori la passione di Celati per il pensatore francese, di cui lui cita anche un libretto dove si parla di spazio, di visione e di pittura, L'occhio e lo spirito. In questo saggio Merleau-Ponty si interroga su una idea di profondità che prescinde dalla visione prospettica. Il legame tra le cose è un enigma perché esse «sono rivali dinanzi al mio sguardo». L'enigma è anche la «loro mutua dipendenza nella loro autonomia» (Merleau-Ponty 1989: 19). Le cose stanno tutte insieme nella profondità, che è una dimensione prima. Da questa profondità secondo Merleau-Ponty emergono le cose, che non sono forme ma materialità a più dimensioni, come per esempio il colore, da non considerare in quanto involucro ma in quanto materia che crea identità, strutture, differenze ecc. Celati traduce le idee di Merleau-Ponty parlando di una esposizione affettiva alla visibilità, per cui anche le abitudini dei luoghi devono aiutarci a capire "come i luoghi vogliono essere visti». Questa implicazione continua del vedente e del veduto va intesa come un dialogo ininterrotto, non come una conquista dell'occhio sul mondo. Così Celati interpreta la perplessità di Palomar-Calvino nell'esigenza 
di trovare un nuovo rapporto con il mondo, e questo rapporto passa attraverso l'abbassamento progressivo della soglia delle aspettative. Io non devo pretendere che il mondo mi riveli un messaggio segreto da decifrare, ma devo mettermi in ascolto del mondo sapendo di esserne parte.

Il libro che si intitola Verso la foce è il risultato dell'impatto con il mondo visivo così come lo ha concepito Luigi Ghirri. È il libro del pensare-immaginare. A cui potremmo aggiungere il camminare. Dunque del camminare-pensareimmaginare. In molti punti del libro ritorna il tema del limite, che viene inteso come limite della visione, cioè della veduta del paesaggio. Si tratta però di un limite che non chiude uno spazio visivo ma che anzi lo apre. Qui sentiamo una presenza di Leopardi che si diffonde in ognuna delle quattro parti del libro e che si innerva sia nell'idea di limite o di linea sia nell'idea di «aperto» che ha una radice in Hölderlin e Rilke. L'aperto, cioè il fuori, ciò che è esterno e non corrisponde alle macchinazioni del pensiero ragionante, ha bisogno del limite e della linea. «La linea d'un campo verde lascia spuntare più lontano la curvatura d'un campo quasi giallo, tagliato da un declivio delle argille. Così l'occhio non è lanciato allo sbaraglio come nella pianura assoluta, dove dopo un po' non si riesce più a distinguere quello che è familiare da quello che è insolito, tutto diventa uguale e ci si stanca» (Celati 1989: 63). Merleau-Ponty ha parlato del «potere costituente» della linea, al di là della condanna espressa dai pittori impressionisti. E cita un pensiero di Paul Klee secondo il quale la linea «rende visibile» (Merleau-Ponty 1989: 52), cioè fa esattamente come dice Celati, offre allo sguardo la possibilità di seguire un percorso verso il lontano senza farlo perdere nell'indifferenziato. Il movimento può essere anche concepito al contrario. Prima c'è «il vuoto che accoglie tutte le cose», e in un secondo tempo l'apertura si restringe su un elemento del paesaggio che chiama da fuori: «Noi siamo guidati da ciò che ci chiama e capiamo solo quello» (Celati 1989: 55). Cioè non possiamo capire lo spazio in sé senza una presenza che ci chiama dallo spazio. Celati dice che questo pensiero se lo porta dietro dallo Zibaldone di Leopardi, la pagina dell'agosto del 1821, dove si parla del piacere che nasce dal contrasto tra finito e indefinito e si fa l'esempio di una campagna «arditamente declive in guisa che la vista in certa lontananza non arrivi alla valle». Cioè l'occhio segue la lontananza senza vedere la linea finale, viene tirato lontano dal piacere della lontananza e non viene chiuso da un limite definitivo. Qualcosa di simile Celati nota a proposito della luce che si effonde all'interno di un'architettura regolare come quella della chiesa di S. Andrea di Mantova disegnata da Leon Battista Alberti. L'occhio non si perde nella luce perché il sistema architettonico la manovra giù dai cassettoni, lungo le arcate, disperdendola nella penombra.

Ma nel presente l'insieme del paesaggio si disgrega, lo spazio perde identità perché viene invaso dalla lebbra dell'indistinto e della modernizzazione. Esiste ancora la possibilità che alcune linee del visibile riescano a tenere il soggetto nello spazio? Al contrario dell'ansia del pieno, che caratterizza ormai ogni 
luogo abitato, il soggetto deve riuscire a trovarsi nel vuoto. La poetica di Ghirri unita all'idea dell'indefinito leopardiano portano alla apparenza della piazza di Pomponesco, che già più volte era stata al centro dell'attenzione del fotografo: "La prospettiva delimitata in fondo da due colonne a ridosso dell'argine, imbuto d'una strada silenziosa con belle case antiche, porta l'occhio verso l'aperto. Là in fondo l'aperto si presenta dietro un orizzonte, facendo sentire l'indistinta lontananza che dà un senso alla nostra collocazione spaziale. Piazza quasi sempre vuota, dove il vuoto si riconosce come l'accogliente, e noi accolti potevamo accorgerci degli altri accolti di passaggio, senza la solita sensazione di fastidio» (Celati 1989: 46). Adesso è l'idea della lontananza ad assumere un ruolo importante, in quanto la mente deve sentire la lontananza se vuole riconquistare un senso dell'esistere. La lontananza indica il rapporto tra il luogo dove ci troviamo, il limite dentro cui stiamo, e un luogo lontano di cui però è necessario sentire la presenza.

Possiamo assumere un'idea che Deleuze spiega a proposito di Spinoza se vogliamo entrare meglio dentro questa logica. L'idea riguarda proprio il limite con cui normalmente un soggetto pensa a se stesso, immaginandosi come unità tenuta insieme da un confine più o meno riconoscibile. Questa è un'idea dell'individuo come essenza, cioè dell'individuo come un pieno contenuto dentro un limite. Ma possiamo definire un ente non in base all'essenza ma in base alle potenzialità (la potenza) con cui l'ente può o non può orientarsi verso l'esterno. Ogni ente tende verso un limite, verso un esterno: questa è la sua potenza. Dunque non c'è un soggetto chiuso da una linea, non c'è una linea che identifica un soggetto e lo rende visibile, ma ogni ente è in movimento verso l'esterno e solo fermando questo movimento possiamo tracciare linee. La linea è semplicemente la forma che assume il soggetto in un determinato momento, ma questa forma si modifica, si espande o si ritrae, a seconda dello stato d'affezione del soggetto. (Deleuze: «Le cose non avrebbero altri limiti oltre quelli stabiliti dalla portata delle loro azioni possibili, o della potenza che possono esprimere» [Deleuze 2007: 134]).

L'immaginazione è la facoltà che muove questa linea. Celati dice: noi acquisiamo consapevolezza della nostra presenza nello spazio proprio perché tendiamo verso quella linea ultima (l'orizzonte) oltre la quale sentiamo la presenza dell'aperto. Ed è questa tensione che ci consente di riscoprire l'essere insieme come condizione di salvezza sia tra gli individui sia tra gli individui e il mondo.

Il discorso che è stato tracciato finora potrebbe essere tradotto in termini artistici se considerando l'opera di Giorgio Morandi. Luigi Ghirri ha fotografato lo studio di Morandi accentuando il rapporto tra linee, luce e colore che sta alla base dei quadri del pittore. Ha esasperato lo spazio ristretto dello studio ripieno di oggetti secondo l'intuizione di Longhi di una visione prospettica dove convivono forma e colore. Ma nello stesso tempo Ghirri ha anche lasciato 
vie di fuga all'immaginazione, cioè a quello che Leopardi chiamava l'indefinito. Questo principio viene enunciato da Ghirri nelle sue lezioni, quando parla della luce uniforme come uccisione dell'immagine e della sua profondità, e vede proprio nel colore il mezzo attraverso il quale la luce può determinare relazioni misteriose (Ghirri 2010:195). Giorgio Messori, introducendo l'album fotografico, nota che nelle tele di Morandi il colore non eclissa la luce, al contrario di quanto avviene nell'arte moderna, ma anzi le dà sostanza, ed è la luce che rende uniche le cose che appaiono nei quadri, quasi sempre oggetti umili nei quali si rivela sia la transitorietà che la completezza dell'esistere (Messori 1992). Ghirri ha fotografato quasi sempre piccole porzioni dell'intero spazio dello studio, esasperando i rapporti di linee che nascono tra gli oggetti quando li si osserva da vicino, e la luce radente li rende fissi, eterni, fa di loro delle «apparenze». Messori insiste anche sul fatto che Morandi è un uomo che ha sempre vissuto in uno spazio ristretto, lo spazio essenziale della cucina, e da questo spazio ristretto, scavando con insistenza dentro gli stessi oggetti, è riuscito a creare una visione del mondo. Il più importante critico d'arte che si è occupato di Morandi, Francesco Arcangeli, ha definito «antimediterranea» la luce che attraversa le tele del pittore, e ha voluto differenziare questa luce da quanto Roberto Longhi aveva voluto vedere in una genealogia morandiana che risale fino a Piero della Francesca e comprende Cézanne e Seurat.

Secondo Arcangeli gli oggetti di Morandi nascono dalla combinazione tra una visione platonica, geometrica e le linee che li definiscono rivelando l'emozione del pennello: "Ogni oggetto sembra anzi nascere davanti a noi, come 'figura', nel punto d'incontro tra un platonismo geometrico d'ordine tutto mentale e il tremito infinito e segreto della visione naturale» (Arcangeli 1981: 55). In questi oggetti si percepisce la presenza della linea, ma si tratta di una linea che vibra nell'atmosfera, anzi rende vibrante l'atmosfera e invita l'occhio a seguire un movimento di sprofondamento nel quotidiano. Morandi è un pittore tonale, cioè un pittore che non crede alla luce solare diretta ma la convoglia nella fluidità interna del colore. Questo nasce da un «sentimento di ambientazione esistenziale». Il concetto di ambientazione corrisponde alla ricerca di Celati nello spazio alienato, in quella stessa pianura Padana che secondo Arcangeli è equidistante dalla follia nordica e dalla solennità mediterranea. La tonalità affettiva del mondo padano si fa sentire anche nelle opere di Morandi, che sembrano del tutto rivolte alla rappresentazione di uno spazio chiuso e protetto. Arcangeli cerca in tutti i modi di sottrarre le nature morte di Morandi a un discorso intimistico o sentimentale, per farne invece opere che parlano di una concezione completa dell'umano nella realtà drammaticamente sconvolta della prima parte del '900. Come direbbe Merleau-Ponty, gli oggetti di Morandi creano movimento "per vibrazione o irraggiamento». Li potremmo definire, sempre con Merleau-Ponty, parte della «carne del mondo» e aggiungere che «ogni carne, e anche quella del mondo, irraggia fuori da se stessa» (MerleauPonty 1989: 56). Se entriamo dentro questa logica, ci rendiamo conto che non esiste più un vuoto dentro il quale siamo dispersi ma tutto riesce a entrare in 
consonanza senza bisogno di ipotizzare la mente come macchina che elabora delle prospettive dentro le quali collocare le cose. Allora potremmo usare per le bottiglie di Morandi la stessa espressione che Celati usa per parlare delle fotografie di Ghirri, degli oggetti banali che spesso vi si incontra, e dire che sono anch'esse «il reperto d'un inconscio esterno» (Celati 2011:68).

Un artista che ha rappresentato quasi solo la disperazione dell'uomo nel vuoto è l'americano Edward Hopper. Gli uomini e le donne di Hopper sembrano a malapena ancorati dentro le abitazioni illuminate dalla luce radente del sole. Questa pittura ha molte affinità con il modo con cui il cinema ha reso comune l'immaginario americano, secondo il quale gli individui si trovano spesso in una condizione di disperata solitudine e di pericolo che deriva dall'incombere dell'esterno. Le dimore di Hopper sono luoghi dentro i quali si tenta di trovare conforto dalla forza devastante del vuoto esterno, che viene osservato da finestre e porte come uno spazio di cui si sente il bisogno. A volte sono luoghi desolati dentro i quali non si trova più traccia del corpo umano. La luce entra solo per illuminare una porzione di muro, come avviene nel quadro Sun in an empty Room (1963). Questo quadro è lo sviluppo di un particolare che si trova in alto a destra in una famosa pala di Piero della Francesca, la Madonna di Senigallia: si vedono delle finestre rettangolari, chiuse da vetri lavorati, dai quali la luce esterna filtra battendo sul muro di fronte. Ecco la descrizione di Roberto Longhi: «a sinistra, all'improvviso, oltre una porta i cui stipiti sono ormai rigature luminose, si addentra una vano dove il sole, filtrato dai battenti invisibili di una finestra a rondelle vitree, dispone, nella penombra, il più raro gioco di lumi che si possa vedere» (Longhi 1974: 444). Longhi guarda con acume all'effetto visivo grazie al quale «il rombo di sole sul muro» interno alla stanza si unisca attraverso «una guida di pulviscolo» alla sorgente luminosa esterna. Si affaccia qui un nuovo modo di visione. In entrambi i casi, la luce che rade il muro fa sentire fisicamente lo spazio, lo rende percepibile, e quindi mette la nostra mente nella condizione di poterlo pensare non in senso astratto.

Il motivo della luce che entra radente da una finestra e divide in due settori lo spazio vuoto di una stanza è per noi interessante perché potrebbe essere all'origine di una delle immagini di Verso la foce, dove si capisce esplicitamente che non si tratta di spazio chiuso o spazio aperto ma di una visione che si forma seguendo ancora una volta una linea che crea intorno a sé la possibilità dello spazio. Celati ha parlato in un saggio («Specie di spazi») della necessità di sentire nello spazio la lontananza, necessità che ancora una volta riconduce a una intuizione leopardiana, cioè al piacere che nasce se il soggetto sente che la sua visione non è piena, composta, compatta, ma contiene una linea di fuga lungo la quale l'occhio si perde senza mai arrivare a una mèta definita.

La pagina di Verso la foce coglie così la pienezza di uno spazio dove ogni cosa sembra trovare una collocazione giusta: «Poco fa, mentre giocavo a flipper, c'è stato un momento di silenzio assoluto in queste campagne. La luce entrando 
da una finestra tagliava l'ambiente in due zone, e l'ombra allungava le gambe delle sedie e dei tavoli. Tutto appariva finalmente compatto, come al riparo dalla solitudine e dall'isolamento, sullo sfondo di questa lunga stanza attraversata da un bancone di linoleum verde. E una foto di calciatori alla parete, la macchina per macinare il caffè, una scatola di plastica piena di palline di chewing gum, orari delle corriere vicino alla porta, tutte queste cose sembravano in salvo dentro un ordine leggero e possibile» (Celati 1989: 128).

Si tratta di una natura morta, ma anche di una fotografia che segue alcune delle idee di Ghirri, secondo il quale dentro lo spazio ridotto della fotografia si doveva sentire anche tutto quanto viene escluso e resta fuori. Qui l'ordine leggero non è prospettico ma viene creato dalla linea diagonale della luce e dell'ombra, come se lo spazio nascesse dalla carne del mondo, cioè da un insieme dentro il quale le singole cose non soffrono né per essere isolate né per essere dominate da una mente ordinatrice superiore.

Nel discorso sullo spazio, Celati si ferma sull'intuizione kantiana secondo la quale tutto ciò che conosciamo dell'esterno è un riflesso che avviene nella nostra coscienza, un fenomeno, un'apparenza (Erscheinung). Lo spazio è sempre in rapporto al punto in cui io sono, al punto relativo che io occupo in un certo momento. Non si dà una idea assoluta di spazio. Celati ricorda i quadri di Friedrich, dove si vedono piccole figure umane che contemplano un orizzonte: «I contemplatori di Friedrich, così assorti davanti al mare o davanti a uno spettacolo della natura, sembrano portare in sé quest'idea nuova e sconvolgente: che gli spazi esterni prendano forma nel pensiero di chi li guarda, secondo come appaiono a chi li guarda, secondo lo stato d'animo di chi li guarda; e che neppure esisterebbero come entità definite se nessuno li guardasse» (Celati 2003: 64).

Il racconto Condizioni di luce sulla via Emilia è l'investigazione intorno a un uomo che vede le cose secondo una intuizione del tutto particolare e privata. Coloro che lo fanno parlare (lo scrittore e l'amico Luciano Capelli) desiderano capire di quale natura è questa visione, anche se è impossibile coglierla pienamente. La figura di Emanuele Menini, il dipintore di insegne, nasce da un insieme di elementi che Celati utilizza per costruire un labile contorno: in lui c'è di sicuro qualcosa di Ghirri, così come c'è qualcosa di Leopardi, e in particolare della figura indefinita di Filippo Ottonieri, un sapiente che come Socrate diffonde un pensiero non codificabile ma calato nella pratica del quotidiano. E forse in Menini, pittore di paesaggi e di insegne, c'è anche la traccia del pittore Giorgio Morandi, con la sua idea di un mondo di oggetti fissi e contemporaneamente in disfacimento, geometrici e vibranti, esatti e fantasmatici. Menini non è un personaggio secondo il significato convenzionale del termine, è un punto di vista sul mondo che viene attorniato da altri punti di vista. Il suo ruolo è di far attrito con le opinioni convenzionali. E di modificarle per quanto gli è possibile. E il suo pensiero si esprime per affermazioni a volte così bizzarre che lui stesso dichiara di non capirle fino in fondo. Celati ha messo in figura le sue idee sul 
pensare-immaginare e ne ha accentuato l'inattualità. Anche le idee di Ghirri vengono così citate tra virgolette, e questo è un modo per renderle ancora più vive e piene di potenzialità.

Il pensiero di Menini sulla luce sembra andare in una direzione diversa da quanto abbiamo visto finora. La luce del territorio padano dove lui abita non gli permette di pensare allo spazio perché si trova in una condizione di disfacimento che non consente il formarsi di un'immagine. Si tratta di luce che si dissolve e che dissolve, «luce scoppiata in disfazione». Mentre Menini ricerca invece una luce capace di fermarsi e di rendere stabili le apparenze. Questa luce e questo spazio sono in un rapporto diretto con le modalità di vita di coloro che vivono in questa specie di grande stanza afosa, la pianura Padana. La ricerca del pittore non è solo estetica ma anche etica: «A Emanuele Menini, in quanto pittore di paesaggi, interessava soprattutto capire come appaiono le cose che stanno ferme, quando sono toccate dalla luce» (Celati 1987a: 47).

Nel discorso sullo spazio Celati si ferma a lungo su Cézanne come artista che ha reso possibile la rappresentazione di uno spazio che si forma direttamente sotto i nostri occhi, uno spazio germinante e vibrante. Celati sottolinea che questo vibrare è capace di attirare l'occhio in quanto lo sguardo non segue una successione lineare ma è «un'ispezione non lineare, è un'incertezza del vedere» (Celati 2003: 83).

L'incertezza del vedere è il punto attorno a cui ruota l'intera meditazione di Menini. Anche per lui vedere lo spazio non è un atto semplice o definibile. Anzi, lo spazio che lo circonda è proprio quello che non consente possibilità di visione. Menini cerca un modo per orientare lo sguardo senza disperderlo nell'insieme convenzionale dello spazio. Come il Palomar di Calvino, per il quale era necessario mettere a fuoco quanto si disperdeva nell'infinita ricchezza del quotidiano, anche Menini vuol trovare una dimensione praticabile del visibile. Nel suo pensiero un ruolo fondamentale è rivestito dall'ombra, considerata come elemento che deve respirare attraverso i colori, grazie all'azione della luce. I colori vivaci del presente «ti fanno dimenticare le ombre e i crepuscoli, e ti rendono stupido, ecco il fatto». Il rapporto tra luce e ombra ha un valore specifico che si collega alle abitudini di vita degli umani. Menini è un pensatore della luce e dello spazio. Capisce la mancanza di un rapporto con l'orizzonte, cioè con il lontano, che condanna i suoi simili all'isolamento e alla ricerca di cose che si consumano nella dimensione piatta del presente. La condizione che Menini cerca è quella di una stabilità attraverso la quale vedere e pensare le cose senza che il disturbo incessante del mondo presente le porti via. Sulle sue fotografie dello studio di Morandi, Ghirri dichiara di averle realizzate pensando all'ossessiva attenzione con cui il pittore fissava pochi oggetti del quotidiano, colorandoli, spostandoli, creando un rapporto di vuoto o di pieno a seconda della loro posizione nello spazio. Ghirri capisce la natura di ombra e di fantasma che caratterizza le nature morte di Morandi. 
La linea che delimita gli oggetti di Morandi porta l'orizzonte lontano dentro la dimensione del domestico e del quotidiano. Così le apparenze usurate riescono a diventare anche visioni del vuoto che ci sembra distante ma in realtà acquista familiarità. Dice Menini. «I corpi nella luce sentono il loro isolamento, e vorrebbero scappar via come lepri». Se è necessario elaborare un pensiero delle ombre è altrettanto necessario elaborare un pensiero dell'orizzonte: «e poi dimmi se col tremore addosso uno può pensare all'orizzonte e aver voglia di vivere in sua compagnia. Impossibile!» (Celati 1987a: 52). Se la luce assoluta illumina i corpi senza consentire un rapporto tra essi e la realtà, solo ritornando a pensare al lontano, cioè all'orizzonte, si può sfuggire al rischio di un pensiero che contempla il mondo in porzioni isolate e non comunicanti tra loro ${ }^{2}$.

L'ultima consapevolezza espressa da Menini è quella che riguarda un pensiero dell'immobilità, cioè di un pensiero che riesce a cogliere il mondo senza imprecisione. Il concetto di immobilità racchiude in sé tutto ciò che si oppone a dispersione, fluttuazione, fuga, disfazione. In un certo senso è una derivazione da quella consistency di cui Calvino non era riuscito a scrivere. Solo che la strada indicata da Calvino aveva portato all'isolamento e alla paura del vuoto, che sono le sindromi di cui soffre Palomar. Menini trova la morte nel momento in cui riesce a vedere l'immobilità, che si incarna in una costruzione isolata in mezzo alla campagna: «E una palazzina in stile geometrile, isolata in mezzo ai campi, che non si scorge bene dalla strada. Per vederla bisogna inoltrarsi in un viottolo e fermarsi davanti ad una siepe dove fioriscono le rose canine. Di lì al mare ci saranno forse venti chilometri, ma quella campagna non annuncia in alcun modo l'affollamento delle aree balneari: è tutta vuota, vi si coltivano pomodori, e più avanti in mezzo ad un campo di grano sorge una solitaria torretta dell'elettricità» (Celati 1987a: 59). Ancora una volta è la scrittura di un'apparenza riconducibile a una fotografia di Ghirri, una fotografia che però non è stata scattata realmente. La scrittura ci prospetta l'ultima immagine che si è creata nella mente di Menini, e che lì è rimasta. Questa immagine potrebbe anche ricordare la disposizione regolare delle nature morte morandiane degli anni cinquanta, dove «la luce frontale ... abolisce le ombre sì che gli oggetti paion posati e sospesi ad un tempo in uno spazio che, intuito su tre dimensioni, è poi astratto in una visione sospesa di effetto bidimensionale» (Arcangeli 1981: 230). Ma al di là della suggestione artistica, mi sembra interessante che Menini muoia proprio osservando un'apparenza che gli consente di oltrepassare la soglia che divide la realtà dalla rappresentazione. Si tratta di un'apparenza oltre la quale si intuisce una serie di altre apparenze. Il pensiero dell'immobilità toglie peso alla morte così come toglie peso al mondo.

2 Per il concetto di apparenza rimando a Belpoliti 2016: LIII. 
Bibliografia

Arcangeli F., 1981, Giorgio Morandi, Torino, Einaudi.

Belpoliti M., 2016, «La letteratura in bilico sull'abisso», in Celati G., Romanzi, cronache e racconti, a cura di N. Palmieri e M. Belpoliti, Milano, Mondadori.

Celati, G., 1984, «Finzioni a cui credere», Alfabeta, VI, 67, p. 13.

Celati, G., 1987a, Quattro novelle sulle apparenze, Milano, Feltrinelli.

Celati, G., 1987b, «Palomar, nella prosa del mondo», Nuova Corrente, 100, p. 227242.

Celati, G., 1989, Verso la foce, Milano, Feltrinelli.

Celati, G., 2003, «Collezione di spazi», il verri, 21, 2003, p. 57-92.

Celati, G., 2011, Conversazioni del vento volatore, Macerata, Quodlibet.

Crary J., 2013, Le tecniche dell'osservatore. Visione e modernità nel XIX secolo, Torino, Einaudi.

Deleuze G., 2007, Cosa può un corpo? Lezioni su Spinoza, Verona, Ombre Corte.

Ghirri L., 2010, Lezioni di fotografia, Macerata, Quodlibet.

Longhi R., 1974, Da Cimabue a Morandi, Milano, Mondadori.

Merleau-Ponty M., 1989, L'occhio e lo spirito, Milano, SE.

Messori G., 1992, "Il pianeta sul tavolo», in Ghirri L., Atelier Morandi, Paris, Contrejour - Bari, Palomar. 